\title{
Distance and mass of the M 104 (Sombrero) group
}

\author{
Igor D. Karachentsev ${ }^{1}$, Lidia N. Makarova ${ }^{1}$, R. Brent Tully ${ }^{2}$, Gagandeep S. Anand ${ }^{2}$, \\ Luca Rizzi ${ }^{3}$, and Edward J. Shaya ${ }^{4}$ \\ 1 Special Astrophysical Observatory, The Russian Academy of Sciences, Nizhnij Arkhyz, Karachai-Cherkessian Republic 369167, \\ Russia \\ e-mail: lidia.makarova@gmail.com \\ 2 Institute for Astronomy, University of Hawaii, 2680 Woodlawn Drive, Honolulu, HI 96822, USA \\ 3 W. M. Keck Observatory, 65-1120 Mamalahoa Hwy, Kamuela, HI 96743, USA \\ 4 Astronomy Department, University of Maryland, College Park, MD 20743, USA
}

Received 14 July 2020 / Accepted 25 August 2020

\begin{abstract}
Aims. Distances and radial velocities of galaxies in the vicinity of the luminous early-type galaxy M 104 (Sombrero) are used to derive its dark matter mass.

Methods. Two dwarf galaxies: UGCA 307 and KKSG 30 situated near M 104 were observed with the Advanced Camera for Surveys on the Hubble Space Telescope. The distances $9.03_{-0.51}^{+0.84} \mathrm{Mpc}$ (UGCA 307) and 9.72 $2_{-0.41}^{+0.44} \mathrm{Mpc}$ (KKSG 30) were determined using the tip of the red giant branch method. These distances are consistent with the dwarf galaxies being satellites of Sombrero.

Results. Using radial velocities and projected separations of UGCA 307, KKSG 30, and a third galaxy with an accurate distance (KKSG 29), as well as 12 other assumed companions with less accurate distances, the total mass of M 104 is estimated to be (1.55 \pm $0.49) \times 10^{13} M_{\odot}$. At the $K$-band luminosity of the Sombrero galaxy of $2.4 \times 10^{11} L_{\odot}$, its total mass-to-luminosity ratio is $M_{T} / L_{K}=$ $(65 \pm 20) M_{\odot} / L_{\odot}$, which is about three times higher than that of luminous bulgeless galaxies.
\end{abstract}

Key words. galaxies: dwarf - galaxies: distances and redshifts - galaxies: photometry - galaxies: individual: M104 galaxies: individual: UGCA 307 - galaxies: individual: KKSG 30

\section{Introduction}

The Local Volume of the Universe amounts to almost a thousand galaxies having distance estimates within $11 \mathrm{Mpc}^{1}$ (Karachentsev \& Nasonova 2013). Near the far edge of this volume at a distance of $9.55 \mathrm{Mpc}$ (McQuinn et al. 2016) there is a bright galaxy M 104 (also known as NGC 4594 or the Sombrero galaxy). With an apparent $K$-band magnitude of $m_{K}=5$ m.0 it has the luminosity of $L_{K} / L_{\odot}=11.32 \mathrm{dex}$, which is four times higher than the luminosity of the Milky Way (10.70 dex) or M31 (10.73 dex). Thanks to its luminosity and, by inference, to its stellar mass the Sombrero is the most outstanding galaxy of the Local Volume.

Over recent years several attempts have been undertaken to determine the total (virial) mass of Sombrero using radial velocities and projected separations of its companions. Estimations of $M_{T} / M_{\odot}$ vary widely: $10.90 \mathrm{dex}$ (Makarov \& Karachentsev 2011), 13.17 dex (Karachentsev \& Nasonova 2013), 13.45 dex (Karachentsev \& Kudrya 2014), and 13.96 dex (Kourkchi \& Tully 2017). The main reason of the scatter in estimates of the total mass is caused by the uncertainty on the gravitational binding of Sombrero with galaxies neighbouring in the projection. The Sombrero galaxy is located near the equator of the Local Supercluster where galaxies are concentrated in the filamental structure, the Virgo Southern Extension (VirgoSE; Tully 1982; Kourkchi \& Tully 2017). Many galaxies in the VirgoSE have radial velocities similar to that of Sombrero, but lie at greater distances typical of the Virgo cluster (15-20 Mpc). At a distance

\footnotetext{
1 http://www.sao.ru/lv/lvgdb
}

of $8 \mathrm{Mpc}$ from the core of the Virgo cluster, the Sombrero galaxy lies at the edge of the zero velocity surface bounding the cluster infall domain, a property shared by other galaxies in the VirgoSE over an extended range in distances.

To reveal the true satellites of Sombrero among the neighbouring galaxies we need to measure their distances, preferably with an error $\Delta D<\sim 1 \mathrm{Mpc}$. At present there is only one galaxy, KKSG 29, in the Sombrero vicinity with an accurately measured distance (9.82 $\pm 0.32 \mathrm{Mpc}$; Karachentsev et al. 2018), determined via the tip of the red giant branch (TRGB) method. This distance places the dwarf galaxy KKSG 29 as a physical satellite of the Sombrero galaxy.

In this work we present measurements of TRGB distances for two more dwarf galaxies, UGCA 307 (or DDO 153) and KKSG 30 (or LEDA 3097708) situated close to Sombrero. The distances of both the galaxies agree well with their belonging to the family of Sombrero satellites. The new distance measurements together with other less reliable distance estimates give us a possibility to make more precise value of the virial (orbital) mass of Sombrero.

\section{TRGB distances to UGCA 307 and KKSG 30}

The dwarf galaxies UGCA $307\left(12^{\mathrm{h}} 53^{\mathrm{m}} 56 \mathrm{~s} .8-12^{\circ} 06^{\prime} 21^{\prime \prime}\right)$ and KKSG $30\left(12^{\mathrm{h}} 37^{\mathrm{m}} 35 \mathrm{~s} \cdot 9-08^{\circ} 52^{\prime} 01^{\prime \prime}\right)$ have apparent $B$ magnitudes of 14.6 and $16^{\mathrm{m}} \cdot 3$, respectively, and projected separations of $\sim 3^{\circ}$ with respect to Sombrero. Their radial velocities in the Local Group rest frame, $731 \mathrm{~km} \mathrm{~s}^{-1}$ (UGCA 307) and $918 \mathrm{~km} \mathrm{~s}^{-1}$ (KKSG 30), are close to the radial velocity 

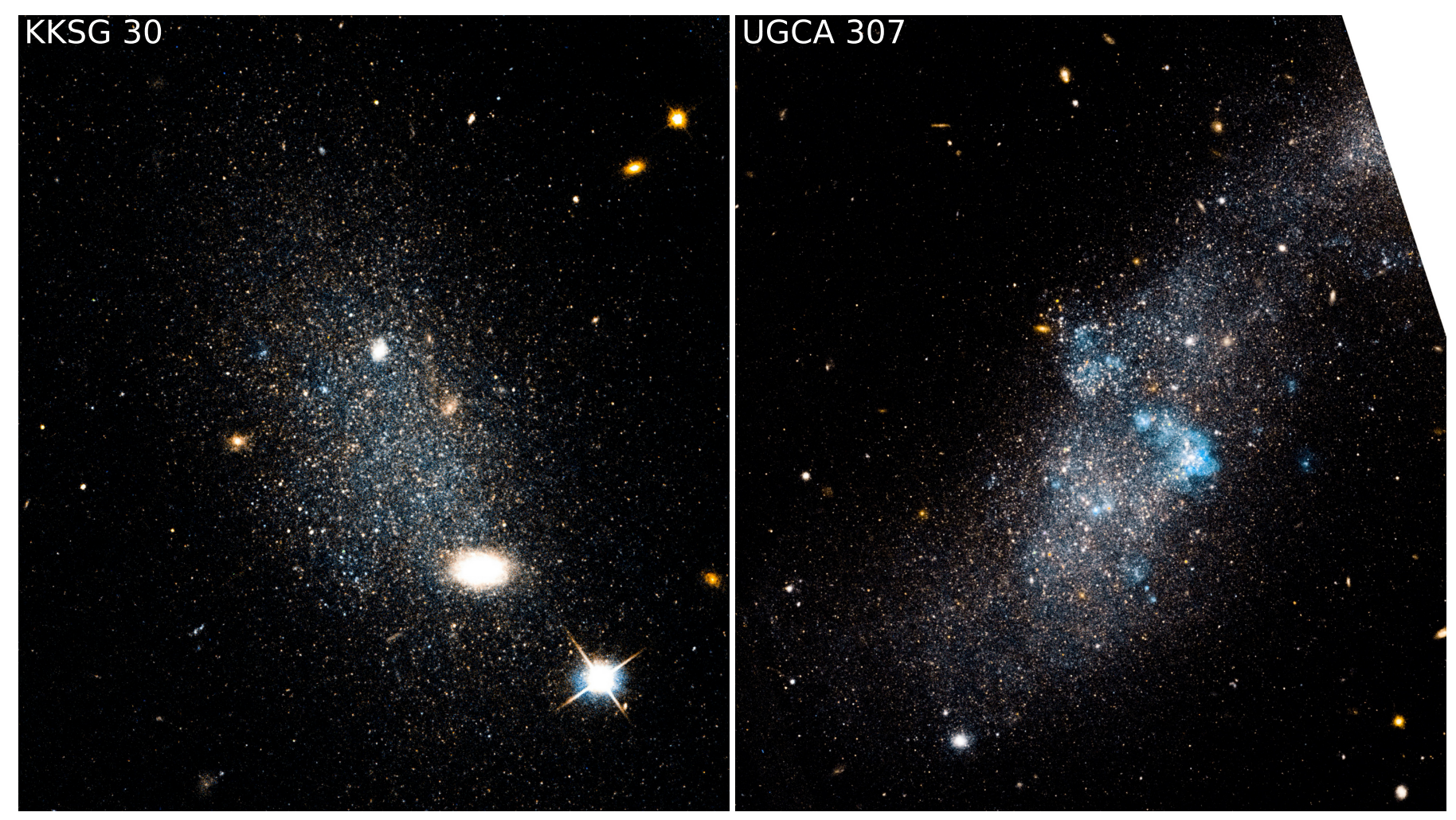

Fig. 1. HST/ACS combined images of UGCA 307 and KKSG 30. The image size is 1:6 $\times 1: 4$. North is up and east is left.

of Sombrero, $892 \mathrm{~km} \mathrm{~s}^{-1}$. The galaxies were observed with the Advanced Camera for Surveys (ACS) aboard the Hubble Space Telescope (HST) on December 5, 2019, and March 13, 2020, as a part of the SNAP project 15922 (PI R.B. Tully).

Two exposures for each object were made in a single orbit with the filters $F 606 W(750 \mathrm{~s})$ and $F 814 W(750 \mathrm{~s})$. The combined images of the galaxies are presented in Fig. 1. We used the ACS module of the DOLPHOT package by Dolphin (2002) to perform photometry of resolved stars based on the recommended recipe and parameters. Only stars with good quality photometry were included in the analysis. We selected the stars with a signal-to-noise ratio $S / N>4$ in both filters, and with DOLPHOT parameters $\operatorname{crowd}_{F 606 W}+\operatorname{crowd}_{F 814 W}<0.8$, $\left(\operatorname{sharp}_{F 606 W}+\operatorname{sharp}_{F 814 W}\right)^{2}<0.075$. Artificial stars were inserted and recovered using the same reduction procedures to accurately estimate photometric errors. Subsequent analysis included only those image regions that contain stars of the galaxies themselves. We selected the region of $1.6 \times 1.6$ arcmin around UGCA 307 and $2.8 \times 1.5$ arcsec around KKSG 30. The resulting colourmagnitude diagrams in $F 606 W-F 814 W$ versus $F 814 W$ are plotted in Fig. 2. A maximum-likelihood method by Makarov et al. (2006) was applied to estimate the magnitude of the TRGB. We found $F 814 W$ (TRGB) to be $25.76_{-0.11}^{+0.19}$ for UGCA 307 and $25.96_{-0.07}^{+0.08}$ for KKSG 30. Following the zero-point calibration of the absolute magnitude of the TRGB developed by Rizzi et al. (2007), we obtained M(TRGB) values of -4.09 (UGCA 307) and -4.08 (KKSG 30). Assuming values of foreground reddening, $E(B-V), 0.049$ (UGCA 307) and 0.028 (KKSG 30) from Schlafly \& Finkbeiner (2011), we derived the true distance modulus of $(m-M)_{0}=29.78_{-0.12}^{+0.20}$ or the distance $D=9.03_{-0.51}^{+0.84} \mathrm{Mpc}$ for UGCA 307 and $(m-M)_{0}=29.94_{-0.09}^{+0.10}$ or the distance $D=9.72_{-0.41}^{+0.44} \mathrm{Mpc}$ for KKSG 30 .

\section{Companions of Sombrero and background objects}

Judging by the big stellar mass of the Sombrero galaxy, the virial radius of its halo can reach about $400 \mathrm{kpc}$. To search for assumed satellites of Sombrero we examined a region of radius $r_{p}=6^{\circ}$ around it that corresponds to the linear projected radius of $R_{p}=1.0 \mathrm{Mpc}$ at the distance of $9.55 \mathrm{Mpc}$. In this area there are 48 galaxies with radial velocities $V_{\mathrm{LG}}<1400 \mathrm{~km} \mathrm{~s}^{-1}$. The data are presented in Table 1 . The table columns contain (1) galaxy name; (2) equatorial coordinates J2000.0; (3) radial velocity in the Local Group rest frame $\left(\mathrm{km} \mathrm{s}^{-1}\right)$; (4) morphological type; (5) apparent $B$ magnitude from the Lyon Extragalactic Database (LEDA, Makarov et al. 2014) or NASA Extra-galactic Database (NED); (6) distance to the galaxy in Mpc; (7) method used for the distance estimate; (8) reference to the source of distance.

As seen from these data, 41 of the 48 galaxies have distance estimates. Most of them were made by the Tully-Fisher method, with uncertainties of 35-30\% for these low luminosity galaxies. Accordingly, we consider only galaxies with distances $D<12 \mathrm{Mpc}$ as probable members of the Sombrero group. The distance and radial velocity distribution of galaxies around Sombrero are given in Fig. 3. In total, 15 galaxies are probable satellites of Sombrero, with the luminosity of each of them more than an order of magnitude fainter than the luminosity of Sombrero. An empty volume (mini-void) is visible ahead of the group. The background galaxies have radial velocities substantially overlapping the Sombrero group velocities. Due to significant TF distance errors, $\Delta D$ of about $3-5 \mathrm{Mpc}$, the membership of some galaxies (UGCA 312, PGC 104868), whether in the group or background may be subject to revision.

The distribution of galaxies from Table 1 in supergalactic coordinates SGL, SGB is presented in Fig. 4. Indications of 

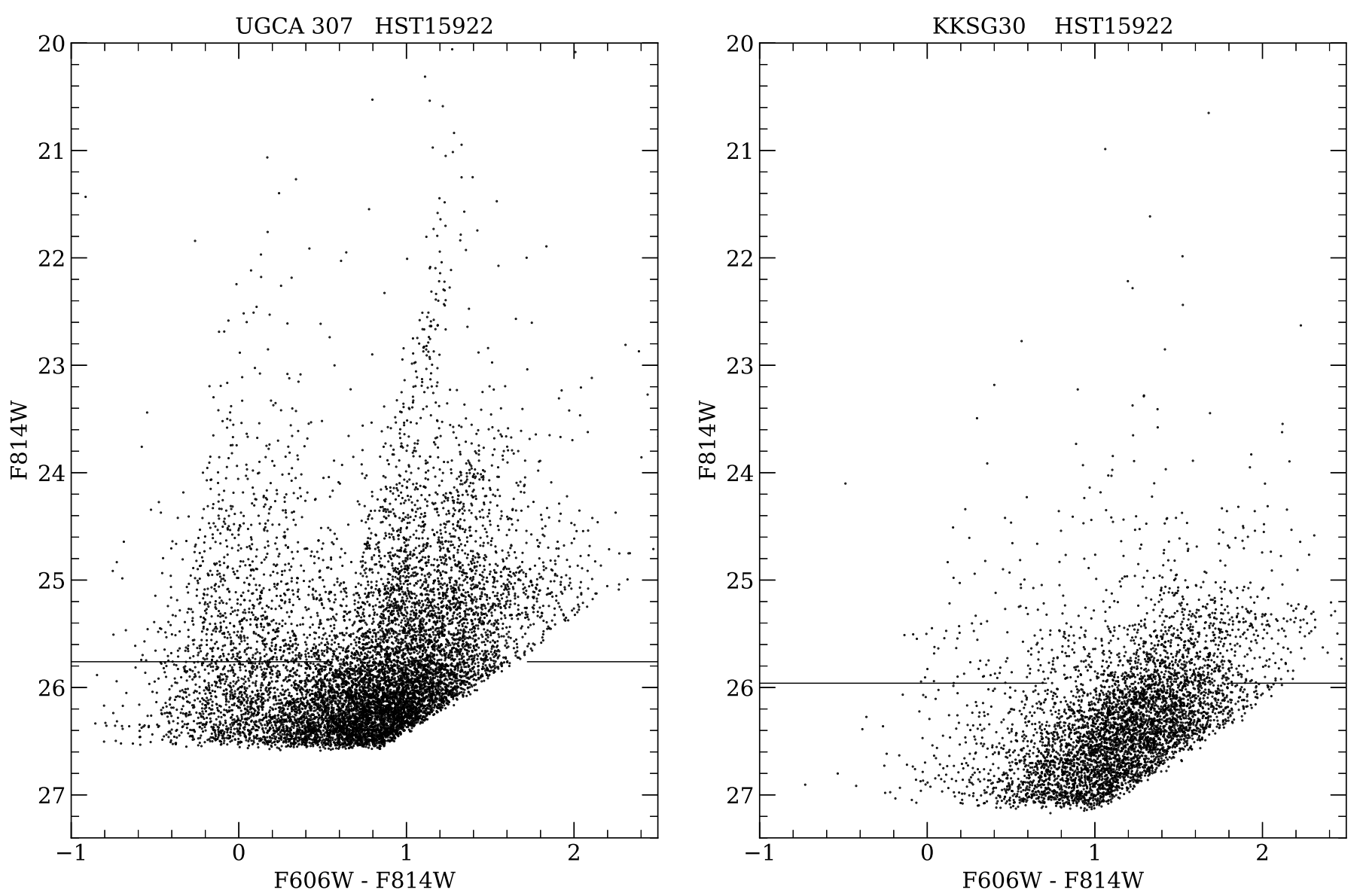

Fig. 2. Colour-magnitude diagrams of UGCA 307 and KKSG 30. The TRGB position is indicated by the horizontal line.

galaxies with different symbols are the same as in the previous figure. Sombrero's satellites, as well as background galaxies, are distributed asymmetrically. In both subsamples there is a noticeable increase in galaxy number towards the supergalactic equator and towards the Virgo cluster centre $\left(\mathrm{SGL}=103^{\circ}, \mathrm{SGB}=-2^{\circ}\right)$. The reason for this asymmetry in the case of Sombrero group members remains unclear to us.

Figure 5 presents the distribution of assumed satellites of Sombrero (solid circles) and background galaxies (open circles) according to angular projected separation, $r_{p}$, and absolute value of radial velocity difference, $|\Delta V|$. Sombrero satellites dominate within $r_{p}<2.4^{\circ}$ (i.e. $400 \mathrm{kpc}$ ), and at larger distances assumed Sombrero satellites are lost among the numerous background galaxies. Such a confusion of two categories of galaxies makes it difficult to estimate the virial mass of the Sombrero galaxy.

\section{Total (orbital) mass of Sombrero}

The list of 15 assumed satellites of Sombrero with known radial velocities is presented in Table 2. The galaxies are ranked according to their projected separation from Sombrero. The average linear projected separations of the satellites is $\left\langle R_{\mathrm{p}}\right\rangle=$ $431 \mathrm{kpc}$, the mean difference of their radial velocities with respect to the principal galaxy is $+62 \pm 54 \mathrm{~km} \mathrm{~s}^{-1}$, and the dispersion of radial velocities is $\sigma_{v}=204 \mathrm{~km} \mathrm{~s}^{-1}$.

We estimated the virial (orbital) mass of the Sombrero galaxy assuming Keplerian motion of satellites, as test particles, around the massive central body. At random orientation of the satellite orbits with the mean eccentricity of $\left\langle e^{2}\right\rangle=1 / 2$
(Barber et al. 2014) the estimate of orbital mass can be written (Karachentsev \& Kudrya 2014) as

$M_{\mathrm{orb}}=(16 / \pi) G^{-1}\left\langle\Delta V^{2} R_{\mathrm{p}}\right\rangle$,

where $G$ is the gravitational constant. If $R_{\mathrm{p}}$ is expressed in kpc and $\Delta V$ expressed in $\mathrm{km} \mathrm{s}^{-1}$, then

$\log \left(M_{\text {orb }} / M_{\odot}\right)=\log \left\langle\Delta V^{2} R_{\mathrm{p}}\right\rangle+6.07$.

Using all 15 assumed satellites from Table 2 we obtain the mean value of orbital mass to be $(17.2 \pm 6.1) \times 10^{12} M_{\odot}$. For three satellites with accurate TRGB distances this quantity is $(15.3 \pm 8.1) \times 10^{12} M_{\odot}$, while for five dwarfs with TRGB and SBF distances the average orbital mass drops to $(10.3 \pm 5.4) \times 10^{12} M_{\odot}$. Attributing to 15 satellite galaxies different weights $(w=1$ for TRGB distances, $w=1 / 2$ for SBF distances and $w=$ $1 / 4$ for TF and mem distances), we derive the weighted mean $(15.5 \pm 4.9) \times 10^{12} M_{\odot}$. We adopt this quantity as the optimal estimate of the total mass of the Sombrero group.

As seen from Table 2, the early-type galaxies are concentrated towards Sombrero much more tightly than spiral and irregular galaxies. Apart from the S0 galaxy NGC 4802, all other Ecompanions reside in the central zone $R_{\mathrm{p}}<200 \mathrm{kpc}$. The known effect of morphological segregation is more pronounced if probable Sombrero satellites without radial velocities are taken into account. Table 3 lists 12 dwarf galaxies of low and very low surface brightness have been detected near Sombrero by different authors (Karachentsev et al. 2000, 2020; Javanmardi et al. 2016; Carlsten et al. 2020a,b). None of them is detected in the 
Table 1. Galaxies around Sombrero with $r_{\mathrm{p}}<6^{\circ}$ and $V_{\mathrm{LG}}<$ $1400 \mathrm{~km} \mathrm{~s}^{-1}$.

\begin{tabular}{|c|c|c|c|c|c|c|c|}
\hline Name & RA Dec & $V_{L G}$ & Type & $B_{T}$ & $D$ & meth. & Ref. \\
\hline$(1)$ & (2) & (3) & (4) & (5) & (6) & (7) & $(8)$ \\
\hline DDO116 & $121628.6-113141$ & 959 & $\mathrm{Sm}$ & 16.02 & 23.28 & $\mathrm{TFb}^{(1)}$ & (1) \\
\hline DDO118 & $121711.9-114041$ & 1064 & Irr & 16.2 & 14.22 & $\mathrm{TFb}$ & (1) \\
\hline PGC104765 & $122143.7-123957$ & 1221 & Irr & 16.92 & & & \\
\hline KKSG27 & $122205.7-094801$ & 1128 & Im & 17.70 & 6.64 & $\mathrm{TFb}$ & (1) \\
\hline UGCA278 & $122310.4-135644$ & 942 & Irr & 16.20 & 18.2 & $\mathrm{TF}$ & (1) \\
\hline PGC970571 & $122549.4-110305$ & 1134 & $\mathrm{BCD}$ & 16.33 & & & \\
\hline PGC157820 & $123001.8-114731$ & 909 & So & 16.13 & & & \\
\hline NGC 4487 & $123104.5-080314$ & 847 & Sc & 11.76 & 20.1 & SN & (2) \\
\hline NGC 4504 & $123217.4-073348$ & 812 & $\mathrm{Sc}$ & 12.12 & 17.5 & $\mathrm{TF}$ & (3) \\
\hline UGCA287 & $123355.4-104047$ & 852 & $\mathrm{Sm}$ & 15.36 & 20.5 & $\mathrm{TF}$ & (4) \\
\hline UGCA289 & $123537.8-075236$ & 800 & Sd & 14.46 & 14.6 & $\mathrm{TF}$ & (3) \\
\hline PGC970397 & $123539.4-110402$ & 928 & Irr & 16.87 & 10.00 & $\mathrm{TF}$ & (1) \\
\hline KKSG29 & $123714.1-102952$ & 562 & Irr & 16.54 & 9.82 & TRGB & (5) \\
\hline KKSG30 & $123735.9-085201$ & 918 & Irr & 16.30 & 9.72 & TRGB & (8) \\
\hline dw1239-1143 & $123915.3-114308$ & 1171 & $\mathrm{dE}$ & 16.80 & 7.9 & SBF & (6) \\
\hline PGC1024539 & $123944.7-070519$ & 744 & Irr & 17.90 & 15.6 & $\mathrm{TFb}$ & (1) \\
\hline NGC 4594 & $123959.4-113723$ & 892 & SOa & 9.00 & 9.55 & TRGB & (7) \\
\hline SUCD1 & $124003.1-114004$ & 1109 & $\mathrm{dE}$ & 18.40 & 9.55 & mem & (1) \\
\hline NGC 4597 & $124012.9-054757$ & 866 & $\mathrm{Sm}$ & 12.87 & 10.10 & $\mathrm{TF}$ & (4) \\
\hline dw1240-1140 & $124017.6-114046$ & 1097 & $\mathrm{dSph}$ & 19.50 & 9.55 & mem & \\
\hline PGC042730 & $124248.9-122327$ & 829 & $\mathrm{dEn}$ & 14.78 & 9.55 & mem & \\
\hline UA295 & $124453.9-090731$ & 1197 & $\mathrm{Sm}$ & 15.10 & 22.9 & $\mathrm{TF}$ & (1) \\
\hline DDO146 & $124541.4-060408$ & 1304 & $\mathrm{Sm}$ & 13.01 & 17.3 & $\mathrm{TF}$ & (3) \\
\hline NGC 4674 & $124603.5-083920$ & 1318 & $\mathrm{Sab}$ & 13.96 & & & \\
\hline PGC1003283 & $124750.9-082816$ & 860 & So & 15.89 & & & \\
\hline PGC104868 & $124854.1-114042$ & 1171 & $\mathrm{BCD}$ & 15.0 & 11.17 & $\mathrm{TF}$ & (1) \\
\hline NGC 4700 & $124908.1-112435$ & 1219 & $\mathrm{Sd}$ & 12.60 & 7.30 & $\mathrm{TF}$ & (4) \\
\hline PGC043345 & $124923.6-100712$ & 1124 & Sdm & 12.79 & 16.6 & $\mathrm{TF}$ & (4) \\
\hline PGC1019240 & $124955.9-072527$ & 1236 & $\mathrm{Sm}$ & 15.52 & & & \\
\hline NGC 4731 & $125101.4-062339$ & 1323 & $\mathrm{SBc}$ & 11.88 & 13.6 & $\mathrm{TF}$ & (3) \\
\hline NGC 4723 & $125103.0-131413$ & 1109 & $\mathrm{Sm}$ & 15.38 & 15.3 & $\mathrm{TF}$ & (4) \\
\hline PGC043526 & $125113.3-063334$ & 1327 & $\mathrm{Im}$ & 13.26 & 13.6 & mem & \\
\hline NGC 4742 & $125148.0-102717$ & 1088 & $\mathrm{E}$ & 12.11 & 15.8 & SBF & (9) \\
\hline NGC 4757 & $125250.1-101836$ & 664 & So & 14.54 & & & \\
\hline UGCA 307 & $125356.8-120621$ & 731 & Im & 14.60 & 9.03 & TRGB & (8) \\
\hline NGC 4781 & $125423.7-103214$ & 1080 & $\mathrm{Scd}$ & 11.39 & 15.5 & $\mathrm{TF}$ & (4) \\
\hline NGC 4790 & $125451.9-101452$ & 1175 & $\mathrm{Sc}$ & 12.41 & 16.9 & $\mathrm{TF}$ & (4) \\
\hline UGCA308 & $125531.1-102350$ & 1140 & Irr & 16.30 & 16.6 & $\mathrm{TFb}$ & (4) \\
\hline NGC 4802 & $125549.6-120319$ & 843 & So & 12.39 & 11.5 & SBF & (10) \\
\hline IC3908 & $125640.6-073346$ & 1127 & Scd & 13.33 & 23.9 & $\mathrm{TF}$ & (4) \\
\hline NGC 4818 & $125648.9-083131$ & 892 & Sab & 11.99 & 11.3 & $\mathrm{TF}$ & (3) \\
\hline UGCA311 & $125746.8-093802$ & 1306 & Scd & 14.00 & 19.8 & $\mathrm{TF}$ & (4) \\
\hline PGC044460 & $125828.3-103437$ & 1173 & Sdm & 14.70 & 8.70 & $\mathrm{TF}$ & (4) \\
\hline UGCA312 & $125906.5-121340$ & 1121 & Irr & 15.88 & 12.0 & $\mathrm{TFb}$ & (4) \\
\hline NGC 4856 & $125921.3-150232$ & 1145 & S0a & 11.44 & 24.0 & $\mathrm{TF}$ & (4) \\
\hline UGCA314 & $130017.2-122048$ & 1397 & $\mathrm{Im}$ & 14.64 & 24.8 & $\mathrm{TF}$ & (4) \\
\hline PGC936912 & $130107.0-133106$ & 1120 & $\mathrm{Im}$ & 15.40 & 14.6 & $\mathrm{TF}$ & (4) \\
\hline NGC 4920 & $130204.2-112243$ & 1155 & $\mathrm{Im}$ & 14.15 & 18.2 & $\mathrm{TF}$ & (4) \\
\hline
\end{tabular}

Notes. (1) Kashibadze et al. (2018), (2) Pejcha \& Prieto (2015), (3) Tully et al. (2016), (4) Karachentsev \& Nasonova (2013), (5) Karachentsev et al. (2018), (6) Carlsten et al. (2020a,b), (7) McQuinn et al. (2016), (8) present paper, (9) Blakeslee et al. (2001), (10) Tonry et al. (2001). (1) "TRGB" - the luminosity of tip of the red giant branch, "SBF" - surface brightness fluctuations, "TF" and "TFb" - the classic Tully \& Fisher (1977) relation or its baryonic version; "SN" - the luminosity of supernova; "mem" - assumed membership in a group with the known distance.

HI line, and all are classified as spheroidal dwarfs. These objects with quenched star formation have projected separations $R_{\mathrm{p}}<$ $200 \mathrm{kpc}$, increasing the segregation effect.

\section{Concluding remarks}

Radial velocities and projected separations of 15 assumed satellites of Sombrero yield the weighted estimate of its total mass $\left(M_{T} / M_{\odot}\right)=(1.55 \pm 0.49) \times 10^{13}$. At $M * / L_{K}=1 M_{\odot} / L_{\odot}$ (Bell et al. 2003) the stellar mass of Sombrero is $2.1 \times 10^{11} M_{\odot}$. Accounting for the luminosity of all the satellites increases

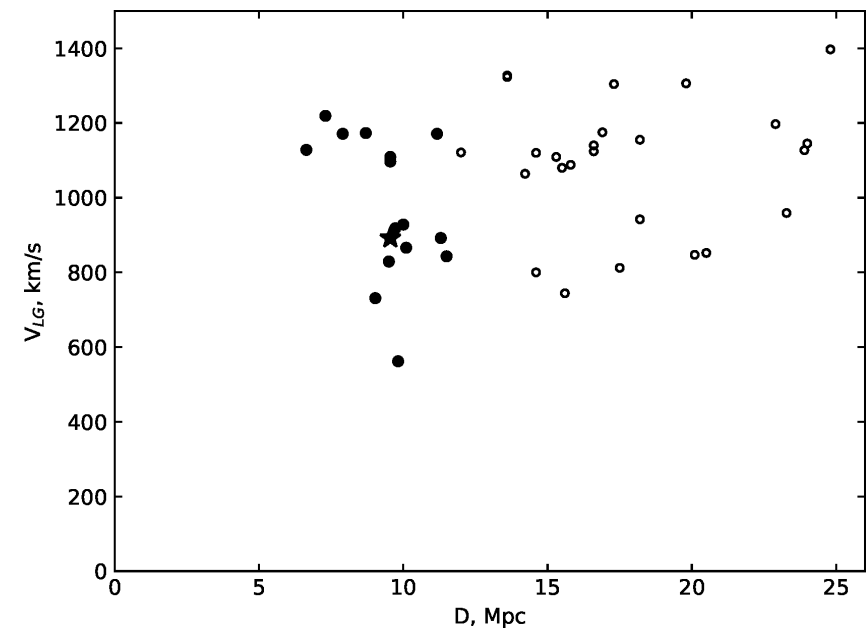

Fig. 3. Distribution of assumed members of the Sombrero group (filled circles) and background galaxies (open circles) according to their distance and radial velocity in the Local Group rest frame. The galaxies satisfy the conditions $V_{\mathrm{LG}}<1400 \mathrm{~km} \mathrm{~s}^{-1}$ and projected separation $r_{\mathrm{p}}<6^{\circ}$ with respect to Sombrero (asterisk).

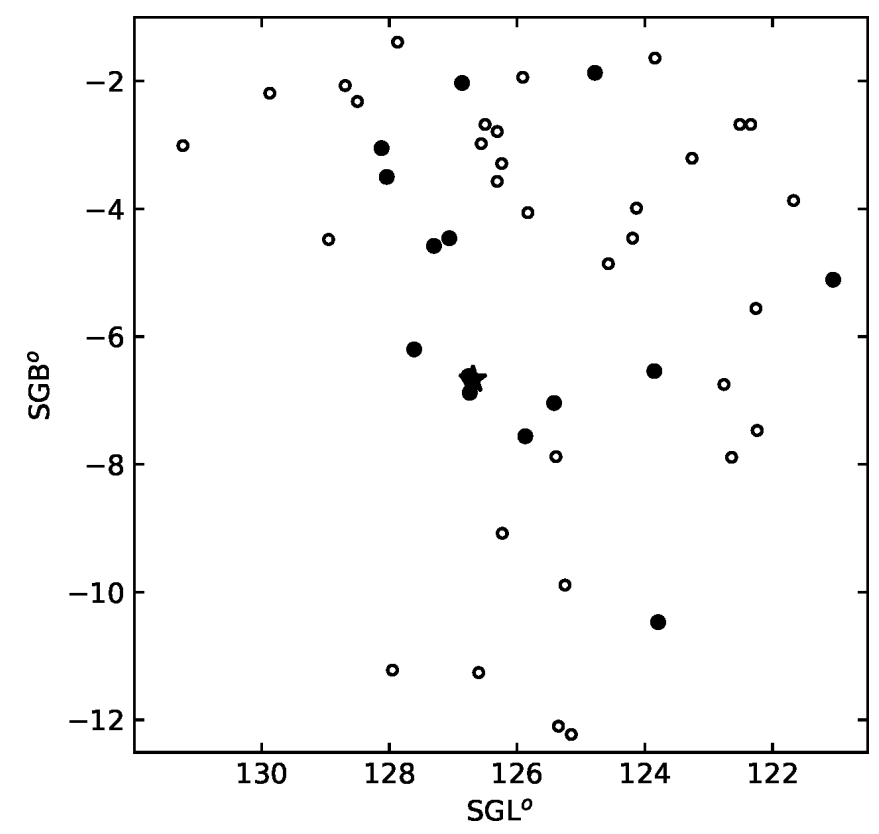

Fig. 4. Distribution of assumed Sombrero satellites (filled circles) and background galaxies (open circles) in supergalactic coordinates. All galaxies have $V_{\mathrm{LG}}<1400 \mathrm{~km} \mathrm{~s}^{-1}$.

the stellar mass of the group to $M *=2.4 \times 10^{11} M_{\odot}$. Therefore, the Sombrero halo has a total-mass-to-stellar-mass ratio of $M_{T} / M *=65 \pm 20$, which is much higher than the cosmic baryonic ratio, $M_{\text {halo }} / M_{b}=6$.

Karachentseva et al. (2011) undertook a search for faint companions around 2MASS Isolated Galaxies (2MIG). They found 214 faint neighbours around 125 2MIG galaxies with radial velocity differences of $|\Delta V|<500 \mathrm{~km} \mathrm{~s}^{-1}$ and projected separations of $R_{\mathrm{p}}<500 \mathrm{kpc}$. For 60 companions around E,S0-galaxies the median ratio of $M_{\text {orb }} / M *$ turns out to be 63, while for the remaining 154 spiral galaxies the median ratio is only 17. A similar search for companions around late-type spiral galaxies without bulges was performed by Karachentsev \& Karachentseva (2019). Based on 43 companions 
I.D. Karachentsev et al.: M 104 (Sombrero)

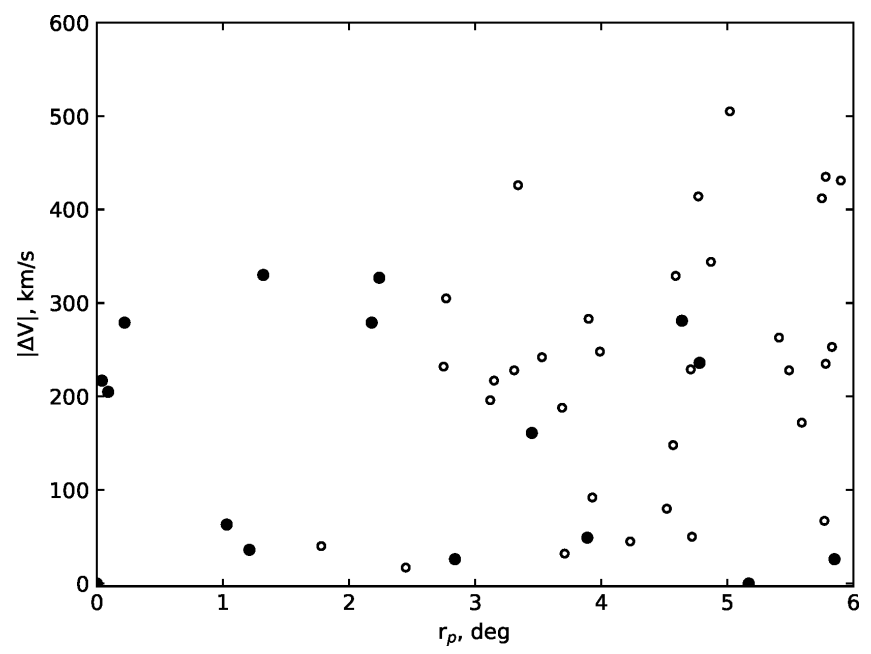

Fig. 5. Radial velocity difference and angular projected separation for assumed members of the Sombrero group (filled circles) and background galaxies (open circles) taken with respect to the Sombrero galaxy.

Table 2. Satellites of Sombrero with known radial velocities.

\begin{tabular}{lccrr}
\hline \hline Name & Type & $r_{\mathrm{p}}$, & $R_{\mathrm{p}}$, & $\Delta V$ \\
\hline SucD1 & & $\left(^{\circ}\right)$ & $(\mathrm{kpc})$ & $\left(\mathrm{km} \mathrm{s}^{-1}\right)$ \\
dw1240-1140 & $\mathrm{dE}$ & 0.04 & 7 & 217 \\
dw1239-1143 & $\mathrm{dE}$ & 0.09 & 15 & 205 \\
PGC042730 & $\mathrm{dE}$ & 1.03 & 171 & 279 \\
PGC970397 & Irr & 1.21 & 201 & -63 \\
KKSG29 & Irr & 1.32 & 220 & -330 \\
PGC104868 & BCD & 2.18 & 362 & 279 \\
NGC 4700 & Sd & 2.24 & 372 & 327 \\
KKSG30 & Irr & 2.84 & 471 & 26 \\
UGCA 307 & Im & 3.45 & 573 & -161 \\
NGC 4802 & S0 & 3.89 & 646 & -49 \\
PGC044460 & Sdm & 4.64 & 770 & 281 \\
KKSG27 & Im & 4.78 & 794 & 236 \\
NGC 4818 & Sab & 5.17 & 858 & 0 \\
NGC 4597 & Sm & 5.85 & 971 & -26 \\
\hline
\end{tabular}

around $30 \mathrm{Sc}$-Scd-Sd galaxies, they found the mean ratio $M_{\text {orb }} / M *=20 \pm 3$. The factor of three difference in halo-massto-stellar-mass ratio between early-type and late-type luminous galaxies attests to their different dynamical histories.

In the vicinity of Sombrero there are still more than a dozen galaxies with unreliable or even unknown distance estimates. Measurements of their TRGB distances with HST would help us to study the structure and dynamics of this group.
Table 3. Assumed satellites of Sombrero without radial velocities.

\begin{tabular}{lcccc}
\hline \hline Name & RA (2000.0) Dec & Type & D, Mpc & meth \\
\hline dw1237-1125 & $123711.6-112559$ & dSph & 7.5 & SBF \\
KKSG31 & $123833.7-102925$ & dSph & 9.55 & mem \\
dw1239-1152 & $123909.0-115237$ & dSph & 8.2 & SBF \\
dw1239-1159 & $123909.1-115912$ & dSph & 11.3 & SBF \\
N4594-DGSAT-3 & $123932.8-111338$ & dSph & 7.9 & SBF \\
Sombrero DwA & $123951.5-112029$ & dSph & 9.7 & SBF \\
KKSG32 & $123955.0-114448$ & dSph & 9.0 & SBF \\
KKSG33 & $124008.9-122153$ & dSph & 9.55 & mem \\
dw1240-1118 & $124009.4-111850$ & dSph & 8.8 & SBF \\
dw1241-1131 & $124102.8-113144$ & dSph & 7.2 & SBF \\
Sombrero DwB & $124112.0-115333$ & dSph & 11.2 & SBF \\
KKSG34 & $124118.9-115539$ & dSph & 9.0 & SBF \\
\hline
\end{tabular}

Acknowledgements. We are grateful to the referee for helpful advice. This work is based on observations made with the NASA/ESA Hubble Space Telescope. STScI is operated by the Association of Universities for Research in Astronomy, Inc. under NASA contract NAS 5-26555. The work in Russia is supported by RFBR grant 18-02-00005.

\section{References}

Bell, E. F., McIntosh, D. H., Katz, N., \& Weinberg, M. D. 2003, ApJS, 149, 289

Barber, C., Starkenburg, E., Navarro, J. F., et al. 2014, MNRAS, 437, 959

Blakeslee, J. P., Lucey, J. R., Barris, B. J., et al. 2001, MNRAS, 327, 1004

Carlsten, S. G., Greene, J. E., \& Peter, A. H. G. 2020a, ApJ, submitted [arXiv:2006.02443]

Carlsten, S. G., Greco, J. P., Beaton, R. L., \& Greene, J. E. 2020b, ApJ, 891, 144 Dolphin, A. E. 2002, MNRAS, 332, 91

Javanmardi, B., Martinez-Delgado, D., Kroupa, P., et al. 2016, A\&A, 588, A89

Karachentseva, V. E., Karachentsev, I. D., \& Melnyk, O. V. 2011, Astrophys. Bull., 66, 389

Karachentsev, I. D., \& Karachentseva, V. E. 2019, MNRAS, 486, 3697

Karachentsev, I. D., \& Kudrya, Y. N. 2014, AJ, 148, 50

Karachentsev, I. D., \& Nasonova, O. G. 2013, MNRAS, 429, 2677

Karachentsev, I. D., Karachentseva, V. E., Suchkov, A. A., \& Grebel, E. K. 2000, A\&AS, 145, 415

Karachentsev, I. D., Makarova, L. N., Tully, R. B., et al. 2018, ApJ, 858, 62

Karachentsev, I. D., Riepe, P., \& Zilch, T. 2020, Ap, 63, 5

Kashibadze, O. G., Karachentsev, I. D., \& Karachentseva, V. E. 2018, Astrophys. Bull., 73, 124

Kourkchi, E., \& Tully, R. B. 2017, ApJ, 843, 16

Makarov, D. I., \& Karachentsev, I. D. 2011, MNRAS, 412, 2498

Makarov, D., Makarova, L., Rizzi, L., et al. 2006, AJ, 132, 2729

Makarov, D. I., Prugniel, P., Terekhova, N., et al. 2014, A\&A, 570, A13

McQuinn, K. B. W., Skillman, E. D., Dolphin, A. E., et al. 2016, AJ, 152, 144

Pejcha, O., \& Prieto, J. L. 2015, ApJ, 799, 215

Rizzi, L., Tully, R. B., Makarov, D., et al. 2007, ApJ, 661, 815

Schlafly, E. F., \& Finkbeiner, D. P. 2011, ApJ, 737, 103

Tonry, J. L., Dressler, A., Blakeslee, J. P., et al. 2001, ApJ, 546, 681

Tully, R. B. 1982, ApJ, 257, 389

Tully, R. B., \& Fisher, J. R. 1977, A\&A, 54, 661

Tully, R. B., Courtois, H. M., \& Sorce, J. G. 2016, AJ, 152, 50 